\title{
Wie sind die Dolomiten zu ihrem Namen gekommen?
}

Eine Marginalie zur Geschichte der Geographie

von Hans-Werner Schütt

Ernst-Günter Stern gewidmet

Die Herkunft des Namens Dolomiten für die als Skigebiet so beliebte Alpengruppe östlich des Etschtals ist zwar, wie ich feststellen konnte, gewöhnlich weder den Touristen noch den Einheimischen bekannt, läßt sich aber, nimmt man nur ein größeres Lexikon zur Hand ${ }^{1}$, schnell klären: die Dolomiten verdanken ihren Namen dem Dolomitgestein und dieses wiederum dem Mineral, aus dem es hauptsächlich gebildet ist, eben dem Dolomit, und das Dolomit-Mineral seinerseits ist benannt nach dem französischen Mineralogen Déodat de Dolomieu, der Ende des 18. Jahrhunderts lebte. Während also die Herkunft der Namen Dolomit und Dolomiten leicht ermittelt werden kann, ist die Frage, wie und warum der Name des genannten Mineralogen auf das Mineral und damit auf das Gestein übergegangen ist, weniger leicht zu beantworten.

Eine Antwort auf dieses Problem ist jedoch durch die Regeln wissenschaftlicher Ethik, wie sie zu Ende des 18. Jahrhunderts allgemein akzeptiert wurden, bereits angedeutet. Es war üblich, daß der Entdecker eines Minerals oder zumindest derjenige, der zuerst eine ausführliche Beschreibung des fraglichen Minerals gegeben hatte, das Recht der Namengebung besaß. Nach dem Vorbild der Biologen ergriffen die Entdecker einer neuen Mineralspezies dabei zuweilen die Gelegenheit, Kollegen zu ehren, die auf gleichem Gebiet Hervorragendes geleistet hatten. Im vorliegenden Fall war derjenige, der das Recht für sich in Anspruch nahm, eine neue Mineralspezies zu benennen, und sie nach Dolomieu benannte, der vor allem wegen seiner pflanzenphysiologischen Arbeiten bekannte Genfer Naturforscher Nicolas-Théodore de Saussure.

Da die Umstände, unter denen es zur Wahl des Namens dolomie oder Dolomit kam, von den Biographien der beiden angesprochenen Forscher abhängig ist, seien diese zunächst einmal kurz vorgestellt.

Dieudonnê-Sylvain-Guy-Tancrède, genannt Déodat, de Gratet de Dolomieu wurde am 23. Juni 1750 in Dolomieu in der Dauphiné als Kind hochadeliger Eltern geboren ${ }^{2}$. Mit zwei Jahren bereits wurde er Novize des Malteser Ordens, in dessen Militärdienst er 1764 eintrat. 1768 tötete er einen Kameraden im Duell, wurde daraufhin gefangengesetzt und erst nach neun Monaten rehabilitiert. Im Jahre 
1775 unternahm er die erste seiner vielen mineralogischen Reisen; er quittierte 1780 den aktiven Militärdienst und widmete sich noch intensiver seinen Studien und seinen Forschungsreisen in West- und Mitteleuropa, die ihn bald zum führenden Vulkanologen seiner Zeit machten. 1783 wurde Dolomieu Kommandant der Truppen auf Malta; Differenzen mit dem Großmeister des Ordens zwangen ihn aber schon bald wieder zum Rücktritt. Nachdem er 1786 auch von der Liste der Kandidaten für den Großen Rat gestrichen worden war, strengte er einen Prozeß vor dem Heiligen Stuhl an, der jedoch nie zu einem definitiven Abschluß führte. Trotz der Parteienkämpfe fand Dolomieu aber noch Zeit zu weiteren mineralogischen Exkursionen. Den Ausbruch der Französischen Revolution begrüßte er lebhaft; 1791 ging er nach Paris und trat dem liberal-großbürgerlichen Club des Feuillants bei, dem viele Naturwissenschaftler angehörten. Nachdem aber Ende 1792 sein Freund und Mentor, der Herzog Alexandre de la Rochefoucauld (17431792), ermordet worden war, wich Dolomieus anfängliche Begeisterung tiefer Skepsis ${ }^{3}$. Obwohl er die Auswüchse der Revolution öffentlich kritisiert hatte, wurde er 1794. zum Professor für Naturgeschichte an der Ecole Centrale ernannt; ein Jahr später wurde er Ingénieur am Corps des Mines. Seit 1795 war Dolomieu Mitglied des Institut National. Trotz seiner Anstellung im Staatsdienst fand er übrigens immer noch Zeit zu Forschungsreisen durch Europa. Im Jahre 1798 nahm Dolomieu an Napoleons Expedition nach Ägypten teil und sah sich, als die französische Flotte vor Malta kreuzte, gegen seinen Willen gezwungen, dem Orden die Kapitulationsbedingungen des Generals zu überbringen. In Ägypten beteiligte er sich an der Erforschung des Landes; doch Krankheit und sein schlechtes Verhältnis zu Napoleon bewogen ihn, sich schon im März 1799 wieder nach Frankreich einzuschiffen. Als sein Schiff vor einem Sturm in Tarent Zuflucht nehmen mußte, wurde er auf Betreiben des Malteser Ordens unter harten Bedingungen in Messina inhaftiert und erst nach der Schlacht bei Marengo 1801 aufgrund des Friedens von Florenz freigelassen. Nach kurzem Aufenthalt in Paris unternahm Dolomieu eine Reise nach Savoyen, auf der er am 16. November 1801 in Châteauneuf bei Clayette (Saône-et-Loire) starb.

So stürmisch wie Dolomieus Leben sich entwickelte, so ruhig scheint das Leben Nicolas-Théodore de Saussures verlaufen zu sein ${ }^{4}$. Am 14.Oktober 1767 als Sohn des Alpenforschers Horace-Bénédict de Saussure (1740-1799) in Genf geboren, war der junge Théodore für eine wissenschaftliche Laufbahn schon dadurch prädestiniert, daß sein Vater ihn selbst unterrichtete und früh zur Mitarbeit an seinen Forschungen heranzog. Nach 1788 begleitete der Sohn seinen Vater auch mehrmals auf Expeditionen in die Alpen (Montblanc, Monte Rosa). Nachdem im Jahre 1794 in Genf die Revolution gesiegt hatte, verweigerte der junge Saussure 
den Eid auf die Verfassung und zog es vor, nach England und Frankreich ins Exil zu gehen, wo er Gelegenheit fand, seine chemischen Kenntnisse zu erweitern. 1797 kehrte er nach Genf zurück und begann hier grundlegende Untersuchungen über den Stoffwechsel der Pflanzen. Dabei führte er ein stilles und zurückgezogenes Leben, selbst nachdem er 1802 zum Honorarprofessor der Mineralogie und Geologie an der Genfer Akademie ernannt worden war. Am 18. April 1845 starb er in seiner Vaterstadt Genf.

Die Beziehungen zwischen Dolomieu und Théodore de Saussure scheinen nur locker gewesen zu sein ${ }^{5}$, obwohl anzunehmen ist, daß sich die beiden Forscher zwischen 1794 und 1797 in Paris begegnet sind. In den von Alfred Lacroix herausgegebenen autobiographischen Papieren Dolomieus findet sich der Name des jüngeren Saussure nicht, und nur ein einziger Brief Dolomieus an Saussure war mir zugänglich.

Enger dagegen waren die Beziehungen zwischen Dolomieu und Horace-Bénédict de Saussure. Die beiden Gelehrten standen in regem Briefwechsel. Allerdings haben sie sich, obwohl Dolomieu bereits seit 1776 Alpenreisen unternommen hatte, erst spät persönlich kennengelernt, nämlich im Sommer 1795 anläßlich eines Besuches des Pariser Mineralogen in Genf ${ }^{6}$. Vermutlich ist es auf den Einfluß Dolomieus zurückzuführen, daß der französische Staat im Jahre 1795/96 Saussure das Angebot machte, neben Dolomieu einen Lehrstuhl an der Ecole Centrale zu übernehmen, und daß das Institut National ebenfalls 1796 in einer Abstimmung die Absicht ausdrückte, Saussure zum Vollmitglied zu machen ${ }^{7}$. Damit sollte der unbestritten führende Alpenforscher seiner Zeit geehrt werden, zu dem Dolomieu, wie er selbst bekannte, im Verhältnis eines Schülers zu seinem Lehrer stand $^{8}$. Im Gefängnis in Messina charakterisierte Dolomieu diesen seinen Lehrer mit der Achtung, die einem großen Manne gebührt:

L'opinion publique, sûrement d'accord, en ce point, avec le jugement de la postérité, a fixé la place de cet homme illustre parmi les premiers physiciens du siècle, et à la tête des géologues. Je ne puis rien ajouter de plus pour sa gloire; mais, pour mon honneur, je puis dire qu'il avait de l'amitié pour moi, qu'il m'a souvent cité avec considération, et qu'il a été flatté des hommages publics que je lui ai rendu. Puissé-je, à mon retour dans le monde, trouver la santé de ce grand homme améliorée, et ne pas avoir à pleurer un savant illustre et un ami $^{9}$.

Und eben dieser Respekt, den Dolomieu zeitlebens für Horace-Bénédict de Saussure empfand, hat vermutlich dazu beigetragen, daß Théodore de Saussure im Jahre 1792 als Bezeichnung für ein neuentdecktes Mineral aus den Tiroler Alpen den Namen des Freundes und Schülers seines Vaters vorschlug. Ganz ohne 
sonstiges eigenes Verdienst um diese Namengebung war Dolomieu allerdings nicht, stammt doch eine erste, wenn auch skizzenhafte Beschreibung des Dolomits, den er pierre calcaire très-peu effervescente nannte, aus seiner Feder. Am 30.Januar 1791 nämlich hatte er seinem Freund und Reisegefährten einer Pyrenäenexpedition, dem Baron Philippe Picot de la Peyrouse (1744-1818), einen Brief geschrieben, der im Juli des gleichen Jahres im Band 39 des Journal de Physique veröffentlicht wurde ${ }^{10}$. Dolomieu berichtet darin von einer Reise, die er achtzehn Monate zuvor in die Tiroler Alpen unternommen hatte ${ }^{11}$, und schreibt, er habe feststellen können, daß die Alpen dort teilweise aus einer bestimmten Steinart bestünden, die früher anscheinend auch als Baumaterial gewisser römischer Statuen verwendet worden sei. Diese Steinart zeichne sich dadurch aus, daß sie beim Übergießen mit Säure nur wenig schäume und sich nur langsam löse und daß sie, wenn man sie mit einem harten Gegenstand schlage, phosphoresziere. Dolomieu knüpft daran die - allerdings nur angedeutete - Vermutung, daß es sich bei dieser Steinart um eine Marmorart mit einem gewissen Gehalt an Phlogiston handle, der für die geringe Lösungstendenz und die Phosphoreszenz verantwortlich sei. Mehr als dies interessiert ihn jedoch die Tatsache, daß, obwohl in der von ihm beschriebenen Gegend noch Porphyr- und Granitgesteine vorkommen, doch die Gipfel der Berge aus der von ihm beschriebenen Marmorart bestehen. Er zieht daraus den - wie er meint - zwingenden Schluß, daß die Marmorart der widerstandsfähigste Stein der Gegend sei, während die härteren Porphyr- und Granitgesteine z.B. wegen der unterschiedlichen thermischen Ausdehnungsfähigkeit ihrer Bestandteile offensichtlich leichter zersetzt würden ${ }^{12}$.

Auf die eben beschriebene, nicht sehr ausführliche Veröffentlichung hin hat Théodore de Saussure dann, wie man aus einem privaten Antwortschreiben Dolomieus vom 31. Oktober $1791^{13}$ entnehmen kann, eine chemische Analyse des fraglichen Gesteins vorgenommen und sich anschließend an Dolomieu gewandt. Dolomieu bemerkt in seinem Antwortbrief, daß die vorgelegte Analyse außerordentlich interessant sei; der Stein sei jetzt vor allem deshalb bemerkenswert, weil Saussure einen bedeutend geringeren Wassergehalt als bei anderen Kalksteinen gefunden habe und andererseits mehr air fixe (Kohlendioxid), als er, Dolomieu, vermutet habe. Er verspricht, weitere Steine für neue Untersuchungen zu schicken. Die anscheinend an ihn gerichtete Frage, ob und wie der eben analysierte Stein zu benennen sei, beantwortet Dolomieu dann wie folgt:

Votre question sur le nom à donner à l'espèce de pierres calcaires, dont l'effervescence est pendant longtemps insensible, m'embarrasse. Je vous dirai d'abord que le baptême vous appartient, puisque c'est vous qui lui avez découvert par 
l'analyse son caractère le plus important, celui qui dépend essentiellement de sa composition, l'absence de l'eau, et sans lequel elle n'aurait pas mérité une dénomination particulière. Si cette pierre n'avait appartenu qu'aux montagnes du Tyrol, vous auriez pu la nommer «tyrolensis», mais elle se trouve dans beaucoup d'autres montagnes; les Alpes suisses, savoyardes, italiennes la possèdent aussi. Quant à moi, Monsieur, j'ai l'intention de me servir du droit que se sont donné les botanistes, lorsqu'ils découvrent une plante nouvelle, d'en faire hommage ou à l'amitié ou à la reconnaissance ou au respect, en lui donnant un nom d'homme. Si j'avais eu le bonheur de faire une découverte importante en lithologie, le nom de M. votre père que j'aurais donné à la nouvelle substance, lui aurait appris ma vénération et ma reconnaissance, pour toutes les lumières qu'il a répandues sur une science qui me fournit mes plus douces occupations. Mais je voudrais que mon hommage fut digne de lui ${ }^{14}$.

Ohne Zweifel haben diese wenigen Zeilen in einem Privatbrief ihrem Autor zu einem der gewaltigsten Denkmäler verholfen, die je die Wissenschaft einem ihrer Vertreter hat setzen können. Ohne Zweifel nämlich trägt ein ganzes Marmorgebirge heute nur deshalb den Namen Dolomieus, weil Théodore de Saussure eben diese Zeilen zum Anlaß nahm, die neue Steinart dolomie zu taufen.

Im März 1792 erschien im Journal de Physique ein Artikel aus der Feder Saussures mit dem Titel: Analyse de la Dolomie ${ }^{15}$. Der Aufsatz beginnt mit den Worten:

L'effervescence lente et presqu'insensible que donnent avec les acides certaines pierres calcaires, est un fait sur lequel M. le commandeur de Dolomieu vient de réveiller l'attention des minéralogistes dans une lettre très-intéressante à M. Picot de la Peyrouse. M. Fleuriau de Bellevue a eu la bonté de me donner au retour de son voyage dans le Tyrol, quelques morceaux de cette substance, jusqu'à présent peu connue, et M. de Dolomieu, à qui je me suis adressé ensuite, m'a envoyé de superbes échantillons de ses principales variétés. Cette pierre mérite à tous égards d'avoir un nom particulier, celui de «pierre calcaire peu effervescente» est indéterminé et impropre. On ne saurait mieux la baptiser, qu'en dérivant son nom de celui du célèbre naturaliste qui nous l'a fait connoître ${ }^{16}$.

Im folgenden gibt Saussure eine genaue Charakterisierung des Dolomits, die er in einige Unterkapitel gliedert, nämlich: Caractères extérieurs de la Dolomie Action du feu sur la Dolomie-Action de l'eau et des acides sur la Dolomie-Action de l'eau et des acides sur la Dolomie calcinée - Analyse de la Dolomie - Examen de la phosphorescence que présentent quelques pierres calcaires par le contact d'un corps chaud - Phosphorescence du Spath-fluor.

Zur Kristallform des Dolomits kann Saussure - wie vor ihm Dolomieu - keine genauen Angaben machen, da er keine gut kristallisierten Stücke vorliegen habe ${ }^{17}$. Dagegen berichtet er von einer chemischen Analyse, die er wie folgt wiedergibt: 
Terre calcaire (Kalkerde, Calciumoxid)

Argile (Alaunerde, Aluminiumoxid)

Magnésie (Talkerde, Magnesiumoxid)

Acide carbonique (Kohlensäure, Kohlendioxid)

Somme

Perte

\begin{tabular}{c}
44,29 grains \\
5,86 « \\
1,40 " \\
$46,10 \quad$ " \\
\hline 98,39 grains \\
$1,61 \quad$ " \\
\hline 100,00 grains $^{18}$
\end{tabular}

Obwohl, wie sich schon bald herausstellte, diese Analyse des Dolomits falsch war, hat der Aufsatz von Théodore de Saussure die Bezeichnung Dolomit für immer in die Mineralogie eingeführt, was dadurch erleichtert wurde, daß Richard Kirwan den Namen Dolomite schon 1794 in die zweite Auflage seines berühmten Lehrbuches Elements of Mineralogy aufnahm ${ }^{19}$.

Der Fehler in der Analyse ist übrigens auf eine etwas merkwürdige Weise zutage getreten. Im Jahre 1801 machte nämlich der englische Privatgelehrte Smithson Tennant (1761-1815) ${ }^{20}$ Düngeversuche mit Kalkarten und stellte dabei fest, daß ein Kalkstein namens Dolomit den Pflanzenwuchs nicht förderte, sondern im Gegenteil behinderte ${ }^{21}$. Aufgrund dieses Ergebnisses bezweifelte Tennant die Angaben Saussures und führte eigene Analysen durch, bei denen er fand, daß Dolomit einen hohen Magnesiumanteil besitzt, nämlich etwa gleich viel Talkerde wie Kalkerde, dagegen so gut wie keine Alaunerde (Tonerde) ${ }^{22}$. Zur Klärung des Problems untersuchte der Nestor der Mineralchemiker, Martin Heinrich Klaproth (1743-1817), im Jahre 1807 den Dolomit noch einmal und bestätigte durch ausgedehnte Untersuchungen von Dolomiten verschiedener Provenienz (St.Gotthard, Apenninen, Kärntner Alpen, antiker Dolomit) im wesentlichen Tennants Ergebnisse $^{23}$. Allerdings war Klaproth mit der gemeinsamen Bezeichnung Dolomit für die von ihm untersuchten Gesteinsarten nicht ganz einverstanden, da sie zwar chemisch gleich seien, aber doch, wie er meinte, aus ganz unterschiedlichen Gebirgsarten mit unterschiedlichen Entstehungsepochen zu stammen schienen. Er schreibt am Schluß seines Aufsatzes:

Endlich gebe ich den Gebirgsforschern anheim, ob die Benennungen Dolomit, nach den Resultaten vorstehender Untersuchungen noch brauchbar bleiben, oder wie derselbe nach den Principien der Geognosie zu fixiren sein mögte; da, dem Ansehn nach, die Steinart von Castelmare sowohl, als auch die der Kärnthenschen Alpen, von einer weit jüngeren Epoche der Entstehung zu sein scheint, als die von Campolongo: worüber indessen Beobachtungen an Ort und Stelle genauer entscheiden müssen ${ }^{24}$. 
Am Namen Dolomit hat die Arbeit Klaproths nichts mehr geändert; sie hat im Gegenteil dazu beigetragen, den Dolomit kristallographisch richtig einzuordnen, nachdem es durch die Analyse de Saussures zunächst zu Irrtümern gekommen war. Ein von Abraham Gottlob Werner (1750-1817) als Bitterspath ${ }^{25}$ klassifiziertes Mineral enthielt, wie sich nun herausstellte, die gleichen Bestandteile wie der Dolomit ${ }^{26}$, lag jedoch im Gegensatz zu diesem in gut ausgebildeten Kristallen vor, nämlich in Rhomboedern. Da aber nach Auffassung der damaligen modernen Mineralogie, als deren Hauptvertreter René-Just Haüy (1743-1822) gelten kann, die kristallographische Form und der chemische Inhalt eindeutig verknüpft sind $^{27}$, war damit auch die Kristallform des Dolomits geklärt. Während Haüy 1801 den Dolomit noch irrtümlich als chaux carbonatée aluminifère bezeichnete, ohne eine Kristallform angeben zu können ${ }^{28}$, führte er im Jahre 1809 in seinem Tableau comparatif einen rhomboedrischen Dolomit auf, der in Deutschland auch Rauthenspath oder Bitterspath genannt werde ${ }^{29}$.

Der Übergang des Namens des nun eindeutig charakterisierten Minerals auf das Gebirge, dessen wesentlichen Bestandteil es ausmacht, ging anscheinend langsam, fast unbemerkt vor sich. Was wir heute die Dolomiten nennen, hieß, wie man anhand einer Serie alter Lexika feststellen kann, bis über die Mitte des 19. Jahrhunderts hinaus Tiroler Alpen oder Cadorische Alpen, und erst um 1875 setzte sich langsam der neue Sprachgebrauch durch, zunächst als dolomitische Alpen oder Südtiroler Dolomitalpen ${ }^{30}$.

Wahrscheinlich ist es vor allem auf die um diese Zeit besonders intensive wissenschaftliche Erforschung der Alpen ${ }^{31}$ zurückzuführen, daß eine Bezeichnung aus der modernen Mineralogie eine alte geographische Bezeichnung schließlich verdrängen konnte.

\section{Anmerkungen}

1 z.B. Meyers Enzyklopädisches Lexikon, Bd.7, Mannheim/Wien/Zürich 1973, S. 58 .

2 Die biographische Skizze stützt sich vor allem auf die Biographie Dolomieus von: Lacroix, A., Déodat Dolomieu. Membre de l'Institut National (1750 1801). Sa vie aventureuse - Sa captivité - Ses œuvres - Sa correspondance. 2 Bde., Paris 1921.

3 In einem Brief an den dänischen Theologieprofessor Frederick Munter vom 15.1.1797 kommt seine Resignation und seine Skepsis in bezug auf die politische Bildungsfähigkeit des Volkes besonders deutlich zum Ausdruck. Vgl. Lacroix, Anm. 2, Bd.2, S.137-139. 
4 Die biographische Skizze stützt sich vor allem auf die Biographien N.-T. de Saussures von: Senn, G., Nicolas-Théodore de Saussure aus Genf, 1767-1845. In: Fueter, E. (Hrsg.), Große Schweizer Forscher, Zürich ${ }^{2}$ 1941, S.183f. Macaire (Vorname nicht verzeichnet), Notice sur la vie et les écrits de Théodore de Saussure, Genf 1845 (40 S., Bibliothèque Universelle de Genève). - Für den letztgenannten Literaturhinweis danke ich Herrn Professor G. de Morsier, Genf.

5 Bei Senn und Macaire sind weder der Dolomit noch Déodat de Dolomieu auch nur erwähnt. Herr Professor de Morsier war so freundlich, darüber hinaus auch die üblichen Schweizer biographischen Handbücher zu konsultieren, doch ohne Erfolg.

6 Aus einem Brief Dolomieus vom 11.7.1795 an seinen Genfer Freund MarcAuguste Pictet (1752-1825) läßt sich ablesen, wie sehr Dolomieu diese Begegnung herbeisehnte. Vgl. Lacroix, Anm.2, Bd.2, S. 75 f. Allerdings fand er de Saussure als bereits schwerkranken Mann vor, was ihn, wie er in einem Brief vom 3. August 1796 an Alexandre Brongniart schreibt, tief erschütterte. Vgl. Lacroix, Anm.2, Bd.2, S.113-115.

7 Beide Vorhaben scheiterten. Die Ecole Centrale dotierte ihre Lehrer so schlecht, daß Dolomieu selbst davon abriet, Saussure trotz seiner Krankheit nach Paris reisen zu lassen (Brief vom 21.8.1796 an M.-A.Pictet, vgl. Lacroix, Anm.2, Bd.2, S.121-124), und das Institut National mußte seinen Beschluß rückgängig machen, weil Saussure kein französischer Staatsbürger war (Brief vom 1.10.1796 an de Saussure, mit Kommentar von Lacroix, Anm.2, Bd.2, S.126).

8 Vgl. Lacroix, Anm.2, Bd.1, S.LXIV.

9 Vgl. Lacroix, Anm.2, Bd.1. S.58f.

10 Dolomieu, Déodat de: Lettre du commandeur Déodat de Dolomieu à M. Picot de la Peyrouse, Membre de plusieurs Académies et Président du District de Toulouse: Sur un genre de pierres calcaires très-peut effervescentes avec les acides, et phosphorescentes par collision. In: Journal de Physique 39, Part II (1791) 3-10, Juillet 1791.

11 Sein Begleiter auf dieser Reise, Fleuriau de Bellevue (1761-1852), war ebenfalls ein naturkundebegeisterter Mann, der auch einige Artikel über dieses Gebiet veröffentlicht hatte.

12 Dolomieu, Anm. 10, S.8.

13 Vgl. Lacroix, Anm.2, Bd.2, S. 24-29.

14 Vgl. Lacroix, Anm.2, Bd.2, S.26. Zu diesen Worten seien noch zwei Anmerkungen am Rande erlaubt. Erstens ist wohl erwähnenswert, daß später tatsächlich ein Mineral nach Horace-Bénédict de Saussure benannt worden ist, und zwar taufte Théodore de Saussure 1806 ein Mineral, das sein Vater als eine Jade-Art bezeichnet hatte, ihm zu Ehren Saussurite. Zweitens sollte zumindest darauf hingewiesen werden, daß Dolomieu selbst 1801 eine mineralogische Nomenklatur gefordert hat, in der Benennungen nach verdienten Wissenschaftlern gewiß keinen Platz mehr gefunden hätten. Diese Nomenklatur sollte man nach seinen Worten so anlegen, daß man eine Spezies 
durch eine Serie von Eigenschaftsbezeichnungen charakterisieren könne, «en les mettant sous une sorte de dépendance les uns des autres, afin de former une chaîne d'idées qui remonte des moindres particularités aux premières généralités, et qui réunisse les notions les plus complexes aux considérations les plus simples». Vgl. Dolomieu, Déodat de, Sur la philosophie minéralogique, et sur l'espèce minéralogique, Paris 1801, S.19.

Saussure, Nicolas-Théodore de, Analyse de la Dolomie. In: Journal de Physique 40, Part I (1792) 161-173, Mars 1793.

16 Saussure, Anm. 15, S.161.

17 Er schreibt: «Les caractères extérieurs de la dolomie ne sont pas toujours assez tranchés et assez constans pour pouvoir servir seuls à la faire reconnoître. Je ne l'ai point encore vue sous une forme cristalline déterminée: le grain salin qu'elle a très-souvent, semble indiquer cependant qu'elle en est susceptible. " Saussure, Anm. 15, S.161f.

18 Saussure, Anm.15, S.167. - In Klammern hinzugefügt sind jeweils die von Klaproth verwendete und die heute übliche deutsche Bezeichnung.

19 Kirwan, Richard, Elements of Mineralogy, 2 Bde., London/Dublin ${ }^{2}$ 1794-96. Passim.

20 Tennant nahm erst 1813 einen Lehrstuhl für Chemie in Cambridge an.

21 Tennant, Smithson, Über die verschiedenen zum Dünger anwendbaren Kalkarten. In: Allgemeines Journal der Chemie (Scherer) 5 (1801) 423-431.

22 Tennant, Anm.21, S. $430 \mathrm{f}$.

23 Klaproth, Martin Heinrich, Beiträge zur chemischen Kenntni $\beta$ der Mineralkörper, Bd.4, Posen/Berlin 1807, S.204-223.

24 Klaproth, Anm.23, S.223.

25 Als Bitterspat werden heute reine $\mathrm{MgCO}_{3}$-Kristalle bezeichnet (Magnesit), während Bitterkalk synonym zu Dolomit verwendet werden kann.

26 Klaproth, Martin Heinrich, Beiträge zur chemischen Kenntniß der Mineralkörper, Bd.4, Posen/Berlin 1807, S.236-238.

27 Vgl. Schütt, Hans-Werner, Über den Einfluß mineralogischer Vorstellungen auf die Entdeckung des Berylliums. In: Rete 2 (1974) 169-182.

28 Haüy, René-Just, Traité de Minéralogie, Bd.2, Paris 1801, S. $173 \mathrm{f}$.

29 Haüy, René-Just, Tableau comparatif des résultats de la cristallographie et de l'analyse chimique, Paris 1809, S. 5.

30 Während Pierers Universal-Conversations-Lexikon (Bd.1, Oberhausen/Leipzig 1875, S.469) lediglich den Namen Cadorische Alpen erwähnt, nennt Meyers Konversations-Lexikon (Bd.1, Leipzig ${ }^{2}$ 1874, S.445) bereits den Namen Südtiroler Dolomitalpen.

31 Um diese Erforschung der Alpen machten sich u. a. die Offiziere GuillaumeHenri Dufour (Schweiz, «Dufourscher Atlas»), Julius von Payer (Österreich) und Karl von Sonklar (Österreich) verdient. 


\section{Summary}

The denotation Dolomites is derived from the name of the French mineralogist Déodat de Dolomieu. The paper traces the way by which the newfound mineral species was baptized:

In 1791 Dolomieu briefly described a mineral, which he had found on one of his travels in the Alps. This prompted the Swiss scientist Théodore de Saussure to analyze the mineral chemically and to ask Dolomieu in a letter, if and how the mineral should be baptized. Thereupon Dolomieu answered that 1 . Saussure had the right to baptize the new mineral as he had found its specific caracteristics and that 2 . in case he himself would have had the right to baptize a new mineral species he would have taken the opportunity to honour Théodore de Saussure's father, Horace Bénédict de Saussure, by naming the mineral after him. This seems to have inspired Théodore de Saussure to name the new mineral dolomie. Even though Smithson Tennant and Martin Heinrich Klaproth soon proved that Saussure's analysis of the dolomite had been wrong, the name of the mineral stayed, and around the middle of the 19th century it even began to be used as denotation of the whole mountain-range which Dolomieu had described in 1791.

Dr. Hans-Werner Schütt

Institut für Geschichte der Naturwissenschaften

D-2 Hamburg 13

Bundesstraße 55 